\title{
Estimation of collision response of virtual objects to arbitrary-shaped real objects
}

\author{
Daeho Lee $^{1}$ and Youngjae Lee ${ }^{2 \mathrm{a})}$ \\ ${ }^{1}$ College of Liberal Arts, Kyung Hee University, \\ 1, Seocheon-dong, Giheung-gu, Yongin-si, Gyeonggi-do, 446-701, Korea \\ ${ }^{2}$ College of Culture and Creative Industry, Jeonju University, \\ 45, Baengma-gil, Wansan-gu, Jeonju, 560-759, Korea \\ a)leeyj@jj.ac.kr
}

Abstract: We propose a novel estimation of collision response between a virtual and an arbitrary-shaped real objects for virtual realities. Collision is detected by using the overlap ratio between objects. A normal vector of a collided plane is estimated by the collided region, and collision response is calculated by the motion vector of the virtual object and the normal vector. For testing of the method, a sensory game using Web camera was implemented. As the results are successful beyond expectation, this method can be effectively utilized for interactive virtual systems.

Keywords: human computer interface, collision response, computer game, virtual reality, computer vision

Classification: Science and engineering for electronics

\section{References}

[1] M. Moore and J. Wihelms, "Collision detection and response for computer animation," ACM SIGGRAPH Computer Graphics, vol. 22, no. 4, pp. 289-298, 1998.

[2] C. O'Sullivan and J. Dingliana, "Real-time collision detection and response using sphere-trees," 15th Spring Conf. on Computer Graphics, pp. 83-92, 1999.

[3] Y. P. Atencio, C. Esperanca, and A. Oliveira, "A collision detection and response scheme for simplified physically based animation," Proc. Computer Graphics and Image Processing, pp. 291-298, 2005.

[4] G. Baciu and W. S. K. Wong, "Image-based techniques in a hybrid collision detector," IEEE Trans. Vis. Comput. Graphics, vol. 9, no. 2, pp. 254$271,2003$.

[5] D. Lee and Y. Park, "Robust vehicle detection based on shadow classification," Proc. Int'l Conf. Pattern Recognition, 3, pp. 1167-1170, 2006. 


\section{Introduction}

A lot of interactive virtual systems, such as games and animations, involve many moving objects. Therefore, the motion of objects should be estimated and the collision and the response should be determined [1,2, 3, 4]. Collision detection and response of modelable objects, say virtual objects, are easily processed using approximated models, such as partial rectangle, circle and sphere data $[1,2,3]$. However, real objects, such as body entities in sensory games, are difficult to model because segmentation is one of the classic and critical problems in computer vision [5]. This paper proposes a novel estimation of collision response between the virtual and real objects; a normal vector of a collided plane is estimated by the collided region, and collision response is easily calculated and fast processed.

\section{Collision detection}

In most collision detection methods, the shape of objects is approximated as small rectangles, circles or spheres. The approximation of arbitrary-shaped objects is very difficult, and the simplified shape could not be correctly represented, especially on complicated boundary. So we use a collision detection method using a ratio of collided region to the virtual object. The collision $(C(o, a))$ is detected as follows:

$$
C(o, a)=\left\{\begin{array}{ll}
\text { true } & \gamma \geq \tau \\
\text { false } & \text { otherwise }
\end{array},\right.
$$

where $o$ and $a$ denote regions of virtual and arbitrary-shaped real objects respectively, $\gamma$ a ratio of the collided region to the virtual object, and $\tau$ a threshold value for collision decision. Noise effects of real objects can be considerably reduced if we use this threshold.

\section{Collision response}

In Fig. 1 (a), let $\overrightarrow{o_{t}}$ be a motion vector of moving virtual object, $\Pi$ a collided plane and $\vec{o}_{t+1}$ a motion vector in the next frame, and the collision is assumed as elastic collision in which total momentum and kinetic energy before and

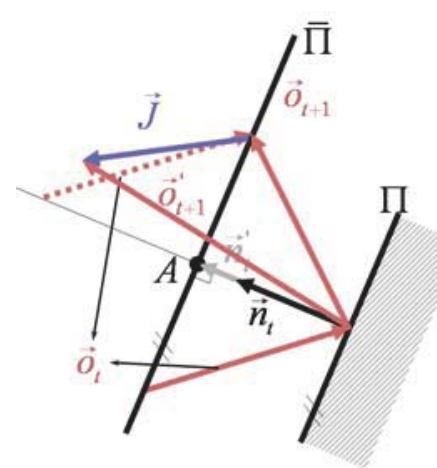

(a) Sphere to plane

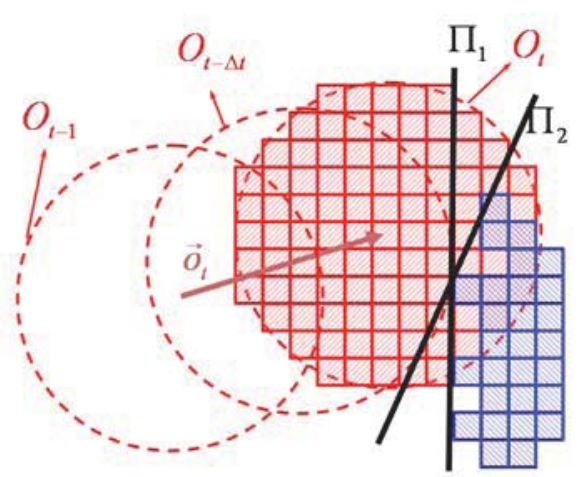

(b) Sphere to arbitrary-shaped object 
after the collision are conserved. Where $\vec{n}_{t}$ is a normal unit vector of $\Pi$, and $\bar{\Pi}$ is a parallel plane of $\Pi$ and is translated to $-\vec{o}_{t}$ with $\vec{n}_{t}$ direction, $A$ is the intersection point of $\bar{\Pi}$ and $\vec{n}_{t}$, and $\vec{n}_{t}^{\prime}$ is a vector which reaches $A$ with $\vec{n}_{t}$ direction. Considering this geometry, $\vec{n}_{t}^{\prime}$ can be calculated by using the inner product of $\vec{o}_{t}$ and $\vec{n}_{t}$, and $\vec{o}_{t+1}$ is the sum of $2 \vec{n}_{t}^{\prime}$ and $\vec{o}_{t}$. So the motion response vector is calculated by

$$
\vec{o}_{t+1}=-2\left(\vec{o}_{t} \cdot \vec{n}_{t}\right) \vec{n}_{t}+\vec{o}_{t}
$$

If the collided plane has its own motion, the motion response may be modified as impulse. Let $\vec{J}$ be an impulse, and the motion response vector is modified as follows:

$$
\vec{o}_{t+1}^{\prime}=\vec{o}_{t+1}+\vec{J}
$$

When a virtual object is a circle, the collision to an arbitrary-shaped real object is illustrated as in Fig. 1(b). The collided plane could not be correctly estimated in digital images and animations, because the motion and the surface are discrete. In Fig. $1(\mathrm{~b})$, let $O_{t}$ and $O_{t-1}$ be the virtual objects in the current $(t)$ and previous frame $(t-1)$, respectively, and the time of collision is $t-\Delta t(0 \leq \Delta t \leq 1)$. Even if the collision position is estimated, the collision plane cannot be determined correctly. That is to say, the collided plane is not decided into $\Pi_{1}, \Pi_{2}$, etc. because of discrete characteristics or noise.

To overcome the problem of collision response, we use a collision region to estimate the normal vector of the collided plane $\left(\vec{n}_{t}\right)$. In Fig. 2, a centroid of the collision region is marked as cross sign, and it can be calculated by the moments as follows:

$$
\begin{aligned}
& m_{x}=\frac{1}{N} \sum_{(x, y, z) \in C} x m(x, y, z), \\
& m_{y}=\frac{1}{N} \sum_{(x, y, z) \in C} y m(x, y, z),
\end{aligned}
$$

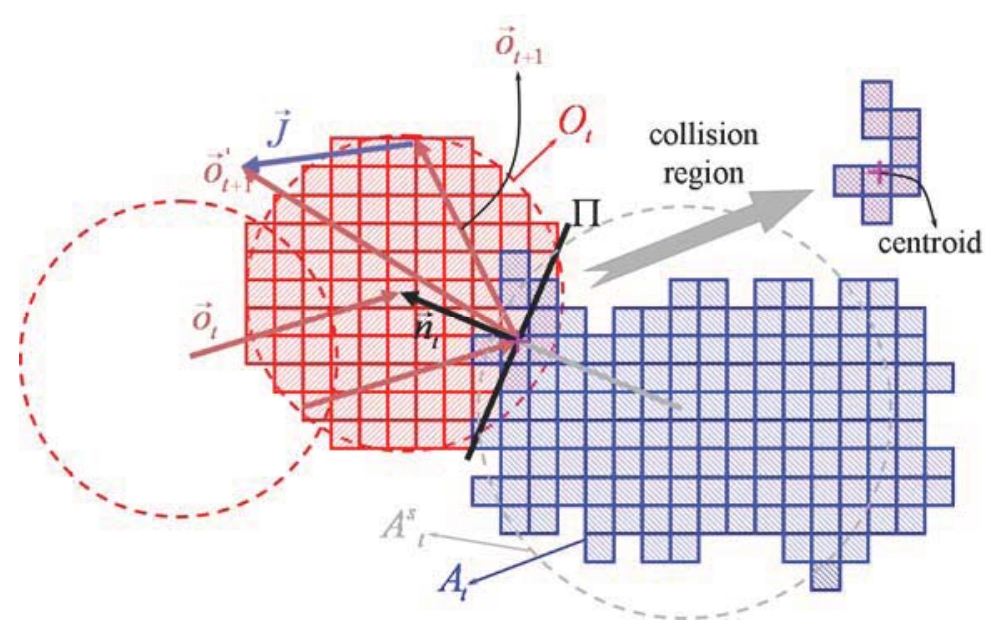

Fig. 2. Collision response for virtual and arbitraryshaped real object. 


$$
m_{z}=\frac{1}{N} \sum_{(x, y, z) \in C} z m(x, y, z)
$$

where $m_{x}, m_{y}$ and $m_{z}$ denote the first order moment of $x, y$ and $z$ axes, respectively, $C$ the collision region, $N$ the number of pixels (voxels) in $C$, and $m(x, y, z)$ mass at $(x, y, z)$. Supposing real objects have uniform mass, $m(x, y, z)$ may become a trivial term. And, the normal vector of the collided plane may be estimated to the vector from the centroid to the center of the virtual object $\left(o_{c}\right)$ as follows:

$$
\vec{n}_{t}=\left(m_{x}, m_{y}, m_{z}\right)-o_{c}
$$

where $\vec{n}_{t}$ is not unit vector.

The motion response can be easily calculated by Eq. (2) and Eq. (3). The proposed estimation of the normal vector can be identified by sphere to sphere collision response. In detail, the centroid of the collision region is regarded as one of the intersections of two spheres $\left(O_{t}\right.$ and $\left.A_{t}^{s}\right)$ as illustrated in Fig. 2. The collision centroid of $O_{t}$ and $A_{t}$ is equal to one of $O_{t}$ and $A_{t}^{s}$, and $A_{t}^{s}$ is the sphere estimated by the collision like $A_{t}$. In sum, the proposed method is similar to sphere to sphere collision, but the sphere model of the real object should be made by estimation of the normal vector.

In addition, one criterion must be utilized; when the included angle between $\vec{o}_{t}$ and $\vec{n}_{t}$ is less than $\pi / 2$, the motion response may be calculated only by impulse because the real object cannot be controlled. This model can be applied to variously shaped virtual objects if these are approximated by partial sphere data.

\section{Experimental result and conclusion}

For testing of the proposed method, a sensory game using Web camera was implemented. Moving body parts (especially arm) are captured by the camera and virtual circle object moves. Frame difference of the body parts is applied to the arbitrary-shaped real object. When the virtual object collides with moving body parts, the collision is detected and the object moves by collision response. Fig. 3 (a) and 3 (b) show consecutive three frames and the results show correct collision detections and responses. In Fig. 3, the red and green arrows represent the motions of virtual object in the current and next frames, respectively, and blue arrows represent the impulse, which is approximated as follows:

$$
\vec{J}=w \vec{n}_{t},
$$

where $w$ denotes the weight of impulse. The yellow regions represent the real objects, which are frame difference. Fig. 3 (c) shows the results when the included angle between $\vec{o}_{t}$ and $\vec{n}_{t}$ is less than $\pi / 2$, so the collision response is estimated only by impulse.

In this paper, the novel method, which is collision response between virtual and arbitrary-shaped real objects for virtual systems, has been presented. The results of testing on Web camera are successful for sensory games. So 


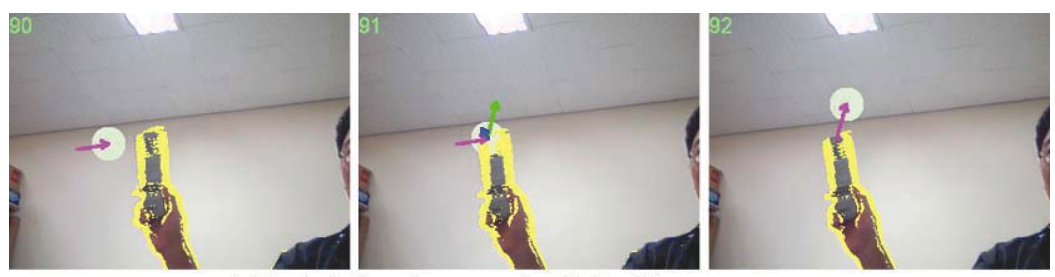

(a) Included angle range: $[\pi / 2,3 \pi / 4)$

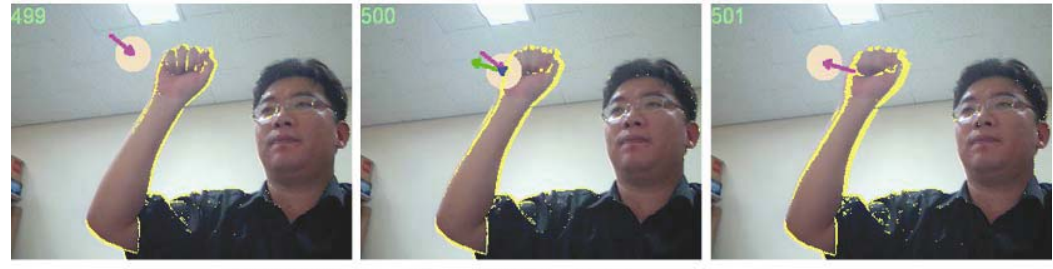

(b) Included angle range: $[3 \pi / 4, \pi)$

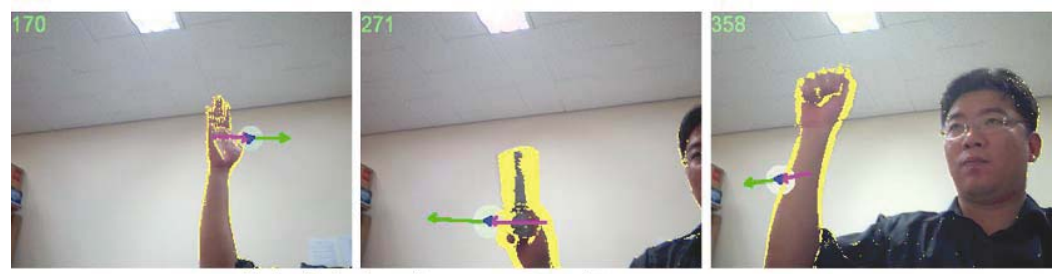

(c) Included angle range: $[0, \pi / 2)$

Fig. 3. Collision response results.

our method can be applied to 3D model, and various virtual objects can be utilized if these are approximated by partial sphere data. Therefore, our method can be utilized effectively for sensory games using computer vision or animation. 